\title{
The Boundedness of Marcinkiewicz Integral Associated with Schrödinger Operator and Its Commutator
}

\author{
Dongxiang Chen and Dan Zou \\ Department of Mathematics, Jiangxi Normal University, Nanchang 330022, China \\ Correspondence should be addressed to Dongxiang Chen; chendx020@yahoo.com.cn
}

Received 7 March 2014; Accepted 20 April 2014; Published 8 May 2014

Academic Editor: Yongsheng S. Han

Copyright ( 2014 D. Chen and D. Zou. This is an open access article distributed under the Creative Commons Attribution License, which permits unrestricted use, distribution, and reproduction in any medium, provided the original work is properly cited.

The authors prove that Marcinkiewicz integral operator is not only are bounded on $L^{p}$, for $1<P<\infty$, but also a bounded map from $L^{1}\left(R^{n}\right)$ to weak $L^{1}\left(R^{n}\right)$. Meanwhile, the $B M O_{L}$-boundedness and $\left(H_{L}^{1}, L^{1}\right)$-boundedness are also obtained. Finally, the $L^{p}$-boundedness and $\left(L^{\infty}, B M O_{L}\right)$-boundedness for the commutator of Marcinkiewicz integral of schrödinger type are established.

\section{Introduction and Notation}

Let us consider the Schrödinger operator

$$
L=-\Delta+V
$$

in $R^{d}, d \geq 3$. The function $V$ is nonnegative, $V \neq 0$, and belongs to a reverse Hölder class $R H_{q}$, for some exponent $q>d / 2$; that is, there exists a constant $C$ such that

$$
\left(\frac{1}{|B|} \int_{B} V(y)^{q} d y\right)^{1 / q} \leq \frac{C}{|B|} \int_{B} V(y) d y
$$

for every ball $B \subset R^{d}$.

We introduce the definition of the reverse Hölder index of $V$ as $q_{0}=\sup \left\{q: V \in R H_{q}\right\}$. It is known that $V \in R H_{q}$ implies $V \in R H_{q+\epsilon}$, for some $\epsilon>0$. Therefore, under the assumption $V \in R H_{d / 2}$, we may conclude $q_{0}>d / 2$. by

The classical Marcinkiewicz integral operator $\mu$ is defined

$$
\mu f(x)=\left(\int_{0}^{\infty}\left|\int_{|x-y| \leq t} \frac{\Omega(x-y)}{|x-y|^{n-1}} f(y) d y\right|^{2} \frac{d t}{t^{3}}\right)^{1 / 2} .
$$

The above operator was introduced by Stein in [1] as an extension of the notion of Marcinkiewicz function from one dimension to higher dimension. Meanwhile, Stein [1] showed that if $\Omega \in \operatorname{Lip}_{\alpha}\left(S^{n-1}\right)$, for some $0<\alpha \leq$ 1 , then $\mu$ is a bounded operator on $L^{p}\left(R^{n}\right)$, for $1<$ $p \leq 2$, and is a bounded map from $L^{1}\left(R^{n}\right)$ to weak $L^{1}\left(R^{n}\right)$. Benedek et al. [2] showed that if $\Omega$ is continuously differentiable in $x \neq 0$, then $\mu$ is a bounded operator on $L^{p}\left(R^{n}\right)$, for $1<p \leq \infty$. Ding et al. [3] proved that the Marcinkiewicz function $\mu$ is bounded from $H^{1}\left(R^{n}\right)$ to $L^{1}\left(R^{n}\right)$ with $\Omega$ satisfying cancelation condition on $S^{n-1}$ and $L^{1}$-Dini condition.

Similar to the classical Marcinkiewicz function $\mu$, we define the Marcinkiewicz integral $\mu_{j}^{L}$ associated with the Schrödinger operator $L$ by

$$
\mu_{j}^{L} f(x)=\left(\int_{0}^{\infty}\left|\int_{|x-y| \leq t} K_{j}^{L}(x, y) f(y) d y\right|^{2} \frac{d t}{t^{3}}\right)^{1 / 2},
$$

where $K_{j}^{L}(x, y)=\widetilde{K_{j}^{L}}(x, y)|x-y|$ and $\widetilde{K_{J}^{L}}(x, y)$ is the kernel of $R_{j}^{L}=\left(\partial / \partial x_{j}\right) L^{-1 / 2}, j=1, \ldots, d$. In particular, when $V=$ $0, K_{j}^{\Delta}(x, y)=\widetilde{K_{j}^{\Delta}}(x, y)|x-y|=\left((x-y)_{j} /|x-y|\right) /|x-y|^{d-1}$ and $\widetilde{K_{j}^{\Delta}}(x, y)$ is the kernel of $R_{j}=\left(\partial / \partial x_{j}\right) \Delta^{-1 / 2}, j=1, \ldots, d$. 
We also give the definition of the commutator generalized by $\mu_{L}^{j}$ and $b$ by

$$
\begin{aligned}
& {\left[b, \mu_{L}^{j}\right](f)(x)} \\
& \quad=\left(\int_{0}^{\infty}\left|\int_{|x-y| \leq t} K_{j}^{L}(x, y)(b(x)-b(y)) f(y) d y\right|^{2} \frac{d t}{t^{3}}\right)^{1 / 2} .
\end{aligned}
$$

In this paper, we write $K_{j}(x, y)=K_{j}^{\Delta}(x, y)$ and

$$
\mu_{j} f(x)=\left(\int_{0}^{\infty}\left|\int_{|x-y| \leq t} K_{j}(x, y) f(y) d y\right|^{2} \frac{d t}{t^{3}}\right)^{1 / 2} .
$$

For a given potential $V \in R H_{q}$, with $q>d / 2$, we introduce the auxiliary function

$$
\rho(x)=\sup \left\{r>0: \frac{1}{r^{d-2}} \int_{B(x, r)} V \leq 1\right\}, \quad x \in R^{d} .
$$

The above assumptions $\rho(x)$ are finite, for all $x \in R^{d}$.

Proposition 1 (see [4]). There exist $C$ and $k_{0} \geq 1$ such that

$$
\begin{aligned}
& C^{-1} \rho(x)\left(1+\frac{|x-y|}{\rho(x)}\right)^{-k_{0}} \\
& \quad \leq \rho(y) \leq C \rho(x)\left(1+\frac{|x-y|}{\rho(x)}\right)^{k_{0} /\left(k_{0}+1\right)},
\end{aligned}
$$

for all $x, y \in R^{d}$.

In particular, $\rho(x) \simeq \rho(y)$, if $|x-y|<C \rho(x)$. A ball $B(x, \rho(x))$ is called critical.

Proposition 2 (see [5]). There exist a sequence of points $\left\{x_{k}\right\}_{k=1}^{\infty}$ in $R^{d}$, so that the family $B_{k}=B\left(x_{k}, \rho\left(x_{k}\right)\right), k \geq 1$, satisfies the following:

(1) $\bigcup_{k} B_{k}=R^{d}$;

(2) there exists $N$ such that, for every $k \in \mathbb{N}$, card $\{j$ : $\left.4 B_{j} \cap 4 B_{k} \neq \varnothing\right\} \leq N$.

Function $f$ is an element of $\mathrm{BMO}_{L}$, if $\exists C>0$ such that, for every ball $B=B(x, r)$,

$$
\int_{B}\left|f-f_{B}\right| \leq C|B|
$$

if $r<\rho(x)$, and

$$
\int_{B}|f| \leq C|B|
$$

if $r \geq \rho(x)$, where $f_{B}=(1 /|B|) \int_{B} f(x) d x$. Let $\|f\|_{\mathrm{BMO}_{L}\left(R^{d}\right)}$ be the smallest $C$ in the inequalities above. It is easy to verify that $\|f\|_{\mathrm{BMO}\left(R^{d}\right)} \leq 2\|f\|_{\mathrm{BMO}_{L}\left(R^{d}\right)}$, for all $f \in \mathrm{BMO}_{L}\left(R^{d}\right)$.
Lemma 3 (see [6]). For any $l>0$, there exists $C_{l}>0$ such that

$$
\begin{gathered}
\left|K_{j}^{L}(x, y)\right| \leq \frac{C_{l}}{(1+(|x-y| / \rho(y)))^{l}} \frac{1}{|x-y|^{d-1}}, \\
\left|K_{j}^{L}(x, y)-K_{j}(x, y)\right| \leq C \frac{\rho(x)}{|x-y|^{d-2}} .
\end{gathered}
$$

Tang and Dong [6] have shown that Marcinkiewicz integral $\mu_{j}^{L}$ is bounded on $L^{p}\left(R^{n}\right)$, for $1<p<\infty$, and are bounded from $L^{1}\left(R^{n}\right)$ to weak $L^{1}\left(R^{n}\right)$. Meanwhile, they also proved that $\mu_{j}^{L}$ are bounded on $\mathrm{BMO}_{L}\left(R^{n}\right)$ and are also mapped from $H_{L}^{1}\left(R^{n}\right)$ to $L^{1}\left(R^{n}\right)$ under the assumption that $K_{j}^{L}$ satisfy the condition in Lemma 3.

Now, we introduce a new BMO-type space of schrödinger operator. Let $\theta>0$; we define the class $\operatorname{BMO}_{\theta}(\rho)$ of locally integrable functions $b$ such that

$$
\frac{1}{|B(x, r)|} \int_{B(x, r)}\left|b(y)-b_{B}\right| d y \leq C\left(1+\frac{r}{\rho(x)}\right)^{\theta},
$$

for all $x \in R^{d}$ and $r>0$, where $b_{B}=(1 /|B|) \int_{B} b(y) d y$. A norm for $b \in \mathrm{BMO}_{\theta}(\rho)$, denoted by $[b]_{\theta}$, is given by the infimum of the constants in the inequalities above. Notice that if we let $\theta=0$, we obtain the John-Nirenberg space BMO.

With the above definition, we define $\operatorname{BMO}_{\infty}(\rho)=$ $\bigcup_{\theta>0} \mathrm{BMO}_{\theta}(\rho)$. Clearly, $\mathrm{BMO} \subset \mathrm{BMO}_{\theta}(\rho) \subset \mathrm{BMO}_{\theta^{\prime}}(\rho)$, for $0<\theta<\theta^{\prime}$, and hence $\mathrm{BMO} \subset \mathrm{BMO}_{\infty}(\rho)$. Moreover, it is in general a larger class.

For $\theta>0$, we denote by $\mathrm{BMO}_{\theta}^{\log }(\rho)$ the set of functions $b$ such that

$$
\frac{1}{|B(x, r)|} \int_{B(x, r)}\left|b(y)-b_{B}\right| d y \leq C \frac{(1+r / \rho(x))^{\theta}}{1+\log ^{+}(\rho(x) / r)},
$$

for all $x \in R^{d}$ and $r>0$. A norm for $b \in \operatorname{BMO}_{\theta}^{\log }(\rho)$, denoted by $\|b\|_{* \log }$, is given by the infimum of the constants in the inequalities above. Correspondingly, we define $\operatorname{BMO}_{\infty}^{\log }(\rho)=$ $\bigcup_{\theta>0} \operatorname{BMO}_{\theta}^{\log }(\rho)$.

Next, we give some information on the Hardy space associated with Schrödinger operator $L$. We say that a function $f \in L^{1}$ is said to belong to $H_{L}^{1}$, if the semigroup maximal operator $T_{L}^{*}$ is bounded on $L^{1}$. The $H_{L}^{1}$ - norm of $f$ is given by $\|f\|_{H_{L}^{1}}=\left\|T_{L}^{*} f\right\|_{L^{1}}$, where $T_{L}^{*} f(x)=\sup _{t>0}\left|T_{t}^{L} f(x)\right|,\left\{T_{t}^{L}\right\}=$ $\left\{e^{-t L}\right\}$ is a semigroup generated by the Schrödinger operator $L$ (see [7]).

Shen [4] gave the following kernel estimate that we needed.

Lemma 4. If $V \in R H_{d}$, then,

(i) for every $N$, there exists a constant $C$ such that

$$
\left|K_{j}^{L}(x, z)\right| \leq \frac{C(1+(|x-z| / \rho(x)))^{-N}}{|x-z|^{d-1}},
$$


(ii) for every $N$ and $0<\delta<\min \left\{1,1-d / q_{0}\right\}$, there exists a constant $C$ such that

$$
\begin{aligned}
& \left|K_{j}^{L}(x, z)-K_{j}^{L}(y, z)\right| \\
& \quad \leq \frac{C|x-y|^{\delta}(1+(|x-z| / \rho(x)))^{-N}}{|x-z|^{d-1+\delta}}
\end{aligned}
$$

$$
\text { where }|x-y|<(2 / 3)|x-z| \text {, }
$$

(iii) if $K$ denotes the $R^{d}$ vector valued kernel of the classical Riesz operator, for every $0<\delta<2-d / q_{0}$, we have

$$
\left|K_{j}^{L}(x, z)-K_{j}(x, z)\right| \leq \frac{C}{|x-z|^{d-1}}\left(\frac{|x-z|}{\rho(z)}\right)^{\delta},
$$

$$
\text { where } K_{j}(x, z)=K(x, z)|x-z| \text {. }
$$

Inspired by [6], we consider the same boundedness of Marcinkiewicz integral whose kernel satisfies Lemma 4 and $L^{p}$-boundedness of its commutator for $1<p<\infty$ and $\left(L^{\infty}, \mathrm{BMO}_{L}\right)$-boundedness.

Theorem 5. The operators $\mu_{j}^{L}$ are bounded on $L^{p}\left(R^{d}\right)$, for $1<$ $p<\infty$, and are bounded from $L^{1}\left(R^{d}\right)$ to weak $L^{1}\left(R^{d}\right)$. On the other hand, $\mu_{j}^{L}$ are bounded on $B M O_{L}\left(R^{d}\right)$ and are bounded from $H_{L}^{1}\left(R^{d}\right)$ to $L^{1}\left(R^{d}\right)$.

In the note, we devote ourselves to establish the following boundedness of the commutators of Marcinkiewicz integral of schrodinger operator type.

Theorem 6. Let $V \in R H_{d}, b \in B M O_{\infty}(\rho)$, and $p_{0}$ such that $1 / p_{0}=\left(1 / q_{0}-1 / d\right)^{+}$, where $q_{0}$ is the reverse Hölder index of $V$; then, for all $f \in L^{p}$, we have

$$
\left\|\left[b, \mu_{j}^{L}\right] f\right\|_{p} \leq C[b]_{\theta}\|f\|_{p}
$$

where $1<p<p_{0}$.

Theorem 7. Let $V \in R H_{d}$ and $b \in B M O_{\infty}(\rho)$; if b satisfies the stronger condition $b \in B M O_{\infty}^{\log }(\rho)$, then $\left[b, \mu_{j}^{L}\right]$ are bounded from $L^{\infty}\left(R^{d}\right)$ to $B M O_{L}\left(R^{d}\right)$.

Throughout this paper, $C$ denotes the constants that are independent of the main parameters involved but whose value may differ from line to line. By $A \simeq B$, we mean that there exists a constant $C>1$ such that $1 / C \leq A / B \leq C$. We use the symbol $A \lesssim B$ to denote that there exists a positive constant $C$ such that $A \leq C B$.

\section{Notation and Preliminaries}

Denote the following maximal functions for $g \in L_{l o c}^{1}\left(R^{d}\right)$, $\alpha>0$, and $x \in R^{d}$ :

$$
M_{\rho, \alpha} g(x)=\sup _{x \in B \in \mathbf{B}_{\rho, \alpha}} \frac{1}{|B|} \int_{B}|g|
$$

$$
M_{\rho, \alpha}^{\sharp} g(x)=\sup _{x \in B \in \mathbf{B}_{\rho, \alpha}} \frac{1}{|B|} \int_{B}\left|g-g_{B}\right|,
$$

where $\mathbf{B}_{\rho, \alpha}=\left\{B(y, r): y \in R^{d}\right.$ and $\left.r \leq \alpha \rho(y)\right\}$.

Lemma 8 (Fefferman-Stein type inequality [8]). For $1<p<$ $\infty$, there exist $\beta$ and $\gamma$ such that if $\left\{Q_{k}\right\}_{k=1}^{\infty}$ is a sequence of balls as that in Proposition 2, then

$$
\begin{aligned}
\int_{R^{d}} \mid & \left.M_{\rho, \beta}(g)\right|^{p} \\
& \lesssim \int_{R^{d}}\left|M_{\rho, \gamma}^{\sharp}(g)\right|^{p}+\sum_{k}\left|Q_{k}\right|\left(\frac{1}{\left|Q_{k}\right|} \int_{2 Q_{k}}|g|\right)^{p},
\end{aligned}
$$

for all $g \in L_{l o c}^{1}\left(R^{d}\right)$.

Lemma 9 (see [9]). Let $\theta>0$ and $1 \leq s<\infty$. If $b \in$ $\mathrm{BMO}_{\theta}(\rho)$, then

$$
\left(\frac{1}{|B|} \int_{B}\left|b-b_{B}\right|^{s}\right)^{1 / s} \leq[b]_{\theta}\left(1+\frac{r}{\rho(x)}\right)^{\theta^{\prime}},
$$

for all $B=B(x, r)$, with $x \in R^{d}$ and $r>0$, where $\theta^{\prime}=\left(k_{0}+1\right) \theta$ and $k_{0}$ is the constant appearing in Proposition 1.

Lemma 10 (see [9]). Let $b \in B M O_{\theta}(\rho), B=B\left(x_{0}, r\right)$, and $s \geq 1$; then

$$
\left(\frac{1}{\left|2^{k} B\right|} \int_{2^{k} B}\left|b-b_{B}\right|^{s}\right)^{1 / s} \lesssim[b]_{\theta} k\left(1+\frac{2^{k} r}{\rho\left(x_{0}\right)}\right)^{\theta^{\prime}},
$$

for all $k \in N$, with $\theta^{\prime}$ as in Lemma 9 .

Next, we introduce our important Lemmas.

We denoted by $M$ the Hardy-Littlewood maximal function and, for $s>1$, by $M_{s}$, the operator is defined as $M_{s}=$ $\left(M\left(f^{s}\right)\right)^{1 / s}$.

Lemma 11. Let $V \in R H_{d}, 1 / p_{0}=\left(1 / q_{0}-1 / d\right)^{+}$, and $b \in$ $B_{M O}(\rho)$. Then, for any $1<s<p_{0}$, there exists a constant $C$ such that

$$
\frac{1}{|Q|} \int_{Q}\left|\left[b, \mu_{j}^{L}\right] f\right| \leq C[b]_{\theta} \inf _{y \in Q} M_{s} f(y),
$$

for all $f \in L_{l o c}^{s}\left(R^{d}\right)$ and every ball $Q=B\left(x_{0}, \rho\left(x_{0}\right)\right)$. 
Proof. Let $f \in L^{p}\left(R^{d}\right)$ and $Q=B\left(x_{0}, \rho\left(x_{0}\right)\right)$. We first observe

$$
\left[b, \mu_{j}^{L}\right] f \leq\left|b-b_{\mathrm{Q}}\right| \mu_{j}^{L} f+\mu_{j}^{L}\left(f\left(b-b_{\mathrm{Q}}\right)\right),
$$

and so we have to deal with the average on $Q$ of each term.

Thanks to Hölder's inequality with $1<s<p_{0}$ and Lemma 10, one has

$$
\begin{aligned}
& \frac{1}{|Q|} \int_{Q}\left|\left(b-b_{Q}\right) \mu_{j}^{L} f\right| \\
& \quad \leq\left(\frac{1}{|Q|} \int_{Q}\left|b-b_{Q}\right|^{\prime}\right)^{1 / s^{\prime}}\left(\frac{1}{|Q|} \int_{Q}\left|\mu_{j}^{L} f\right|^{s}\right)^{1 / s} \\
& \quad \leq[b]_{\theta}\left(\frac{1}{|Q|} \int_{Q}\left|\mu_{j}^{L} f\right|^{s}\right)^{1 / s} .
\end{aligned}
$$

Let $f=f_{1}+f_{2}$ with $f_{1}=f \chi_{2 \mathrm{Q}}$; then $L^{s}\left(R^{d}\right)$-boundedness of $\mu_{j}^{L}$, for $1<s<p_{0}$, says that

$$
\begin{aligned}
\left(\frac{1}{|Q|} \int_{Q}\left|\mu_{j}^{L} f_{1}\right|^{s}\right)^{1 / s} & \leqslant\left(\frac{1}{|Q|} \int_{2 Q}|f|^{s}\right)^{1 / s} \\
& \leq \inf _{y \in Q} M_{s} f(y) .
\end{aligned}
$$

Now, for $x \in Q, \rho(x)=\rho\left(x_{0}\right)$ and using Lemma 4 and Minkowski's inequality, we get

$$
\begin{aligned}
\left|\mu_{j}^{L} f_{2}(x)\right| & =\left(\int_{0}^{\infty}\left|\int_{|x-y| \leq t} K_{j}^{L}(x, y) f_{2}(y) d y\right|^{2} \frac{d t}{t^{3}}\right)^{1 / 2} \\
& \leqslant\left(\int_{0}^{\infty} \mid \int_{|x-y| \leq t} \frac{(1+(|x-y| / \rho(x)))^{-N}}{|x-y|^{d-1}}\right. \\
& \left.\times\left.\left|f_{2}(y)\right| d y\right|^{2} \frac{d t}{t^{3}}\right)^{1 / 2} \\
& \leqslant \int_{R^{d}} \frac{\left|f_{2}(y)\right| \rho\left(x_{0}\right)^{N}}{|x-y|^{d-1+N}\left(\int_{|x-y| \leq t} \frac{d t}{t^{3}}\right)^{1 / 2} d y} \\
& \leqslant \rho\left(x_{0}\right)^{N} \int_{R^{d}} \frac{\left|f_{2}(y)\right|}{|x-y|^{d+N} d y} \\
& \leqslant \rho\left(x_{0}\right)^{N} \int_{(2 Q)^{c}} \frac{|f(y)|}{|x-y|^{d+N}} d y \\
& \leqslant \rho\left(x_{0}\right)^{N} \sum_{k=1}^{\infty} \int_{2^{k+1} Q \mid 2^{k} Q} \frac{|f(y)|}{|x-y|^{d+N}} d y \\
& \leqslant \rho\left(x_{0}\right)^{N} \sum_{k=1}^{\infty} \frac{\left(2^{k} \rho\left(x_{0}\right)\right)^{-N}}{\left|2^{k+1} Q\right|} \int_{2^{k+1} Q}^{|f(y)| d y} \\
& \leqslant \inf _{y \in Q} M_{s} f(y) .
\end{aligned}
$$

For the second term, we split again $f=f_{1}+f_{2}$. By choosing $1<\widetilde{s}<s<p_{0}$ and denoting $v=s \widetilde{s} /(s-\widetilde{s})$, by the boundedness of $\mu_{j}^{L}$ on $L^{\tilde{s}}\left(R^{d}\right)$ and Hölder's inequality, we obtain

$$
\begin{aligned}
& \frac{1}{|Q|} \int_{Q}\left|\mu_{j}^{L}\left(f_{1}\left(b-b_{B}\right)\right)\right| \\
& \quad \leq\left(\frac{1}{|Q|} \int_{Q}\left|\mu_{j}^{L}\left(f_{1}\left(b-b_{B}\right)\right)\right|^{\tilde{s}}\right)^{1 / \tilde{s}} \\
& \quad \leq\left(\frac{1}{|Q|} \int_{2 Q}\left|f\left(b-b_{B}\right)\right|^{\tilde{s}}\right)^{1 / \widetilde{s}} \\
& \quad \leq\left(\frac{1}{|Q|} \int_{2 Q}|f|^{s}\right)^{1 / s}\left(\frac{1}{|Q|} \int_{2 Q}\left|b-b_{B}\right|^{\nu}\right)^{1 / v} \\
& \quad \leq[b]_{\theta} \inf _{y \in Q} M_{s} f(y),
\end{aligned}
$$

where, in the last inequality, we have used Lemma 9.

For the remaining term, let $1=1 / s+1 / \widetilde{s}, \rho(x) \bumpeq \rho\left(x_{0}\right)$ using Lemma 9, Lemma 10, and Minkowski's inequality, we arrive to

$$
\begin{aligned}
& \left|\mu_{j}^{L}\left(f_{2}\left(b-b_{\mathrm{Q}}\right)\right)(x)\right| \\
& =\left(\int_{0}^{\infty}\left|\int_{|x-y| \leq t} K_{j}^{L}(x, y) f_{2}(y)\left(b(y)-b_{\mathrm{Q}}\right) d y\right|^{2} \frac{d t}{t^{3}}\right)^{1 / 2} \\
& \leq\left(\int_{0}^{\infty}\left|\int_{|x-y| \leq t} \frac{\left|f_{2}(y)\right|\left|b(y)-b_{\mathrm{Q}}\right|}{|x-y|^{d-1}(1+(|x-y| / \rho(x)))^{N}} d y\right|^{2} \frac{d t}{t^{3}}\right)^{1 / 2} \\
& \leq \int_{R^{d}} \frac{\left|f_{2}(y)\right|\left|b(y)-b_{\mathrm{Q}}\right|}{|x-y|^{d-1}(1+(|x-y| / \rho(x)))^{N}} \\
& \quad \times\left(\int_{|x-y| \leq t} \frac{d t}{t^{3}}\right)^{1 / 2} d y \\
& \leq \rho\left(x_{0}\right)^{N} \int_{(2 \mathrm{Q})^{c}} \frac{|f(y)|\left|b(y)-b_{\mathrm{Q}}\right|}{|x-y|^{d+N}} d y \\
& \leq \rho\left(x_{0}\right)^{N} \sum_{k=1}^{\infty} \frac{\left(2^{k} \rho\left(x_{0}\right)\right)^{-N}}{\left|2^{k+1} \mathrm{Q}\right|} \\
& \quad \times \int_{2^{k+1} \mathrm{Q} \mid 2^{k} \mathrm{Q}}^{|f(y)|\left|b(y)-b_{\mathrm{Q}}\right| d y} \\
& \leqslant \sum_{k=1}^{\infty} \frac{2^{-k N}}{2^{k+1} \mathrm{Q} \mid} \int_{2^{k+1} \mathrm{Q}}^{|f(y)|\left|b(y)-b_{\mathrm{Q}}\right| d y}
\end{aligned}
$$




$$
\begin{aligned}
& \leq \sum_{k=1}^{\infty} 2^{-k N}\left(\frac{1}{\left|2^{k+1} Q\right|} \int_{2^{k+1} Q}\left|b(y)-b_{\mathrm{Q}}\right|^{s^{\prime}} d y\right)^{1 / s^{\prime}} \\
& \times\left(\frac{1}{\left|2^{k+1} Q\right|} \int_{2^{k+1} Q}|f(y)|^{s} d y\right)^{1 / s} \\
& \leq \sum_{k=1}^{\infty} 2^{-k N} k\left(1+\frac{2^{k} \rho\left(x_{0}\right)}{\rho\left(x_{0}\right)}\right)^{\theta^{\prime}}[b]_{\theta} \inf _{y \in \mathrm{Q}} M_{s} f(y) \\
& \leq \sum_{k=1}^{\infty} k 2^{k\left(-N+\theta^{\prime}\right)}[b]_{\theta} \inf _{y \in Q} M_{s} f(y) \\
& \leq[b]_{\theta} \inf _{y \in \mathrm{Q}} M_{s} f(y) .
\end{aligned}
$$

Since $N$ can be chosen large enough, the last series converges. Thus, we finished the proof.

Remark 12. It is easy to check that if the critical ball $Q$ is replaced by $2 Q$, the above lemma also holds.

Lemma 13. Let $V \in R H_{d}$ and $b \in B M O_{\infty}(\rho)$, then, for any $1<s<p_{0}$ and $\gamma \geq 1$, there exists a constant $C$ such that

$$
\begin{aligned}
& \left|\mu_{j}^{L}\left(f_{2}\left(b-b_{B}\right)\right)(u)-\mu_{j}^{L}\left(f_{2}\left(b-b_{B}\right)\right)(z)\right| \\
& \quad \leq[b]_{\theta} \inf _{x \in B} M_{s} f(x),
\end{aligned}
$$

for all $f, u, z \in B=B\left(x_{0}, r\right)$, with $r<\gamma \rho\left(x_{0}\right)$ and $f_{2}$ as in Lemma 11.

Proof. We write

$$
\begin{aligned}
& \left|\mu_{j}^{L}\left(f_{2}\left(b-b_{B}\right)\right)(u)-\mu_{j}^{L}\left(f_{2}\left(b-b_{B}\right)\right)(z)\right| \\
& \leq\left(\int_{|u-y| \leq t<|z-y|}^{\infty} \mid K_{j}^{L}(u, y)\right. \\
& \left.\quad \times f_{2}(y)\left(b(y)-b_{B}\right)|d y|^{2} \frac{d t}{t^{3}}\right)^{1 / 2} \\
& +\left(\int_{0}^{\infty}\left|\int_{|z-y| \leq t<|u-y|}\right| K_{j}^{L}(z, y)\right. \\
& \left.\quad \times f_{2}(y)\left(b(y)-b_{B}\right)|d y|^{2} \frac{d t}{t^{3}}\right)^{1 / 2} \\
& +\left(\int_{0}^{\infty}\left|\int_{\{|u-y| \leq t,|z-y| \leq t\}}\right| K_{j}^{L}(u, y)-\left.K_{j}^{L}(z, y)\right|^{2}\right. \\
& \left.\times\left.\left|f_{2}(y)\left(b(y)-b_{B}\right)\right| d y\right|^{2} \frac{d t}{t^{3}}\right)^{1 / 2} \\
& =I_{1}+I_{2}+I_{3} . \quad
\end{aligned}
$$

Since the estimates for $I_{1}$ and $I_{2}$ follow along similar lines, we only consider $I_{1}$. By denoting $Q=B\left(x_{0}, \gamma \rho\left(x_{0}\right)\right)$, since in our situation $\rho(u)=\rho\left(x_{0}\right)$ and $|u-y|=|z-y|$, by Minkowski's inequality and Lemma 4 , we have

$$
\begin{aligned}
I_{1} \leq & \int_{R^{d}}\left|f_{2}(y)\right|\left|b(y)-b_{B}\right|\left|K_{j}^{L}(u, y)\right| \\
& \times\left(\int_{|u-y| \leq t<|z-y|} \frac{d t}{t^{3}}\right)^{1 / 2} d y \\
\leq & r^{1 / 2} \int_{(2 B)^{c}} \frac{|f(y)|\left|b(y)-b_{B}\right|\left|K_{j}^{L}(u, y)\right|}{|u-y|^{3 / 2}} d y \\
\leq & r^{1 / 2} \int_{Q \mid 2 B} \frac{|f(y)|\left|b(y)-b_{B}\right|}{|u-y|^{d+1 / 2}} d y \\
& +r^{1 / 2} \rho\left(x_{0}\right)^{N} \int_{Q^{c}} \frac{|f(y)|\left|b(y)-b_{B}\right|}{|u-y|^{d+1 / 2+N}} d y \\
= & I_{11}+I_{12} .
\end{aligned}
$$

Splitting into annuli, we have

$$
I_{11} \lesssim \frac{1}{r^{d}} \sum_{j=2}^{j_{0}} 2^{-j(d+1 / 2)} \int_{2^{j} B}|f(y)|\left|b(y)-b_{B}\right| d y,
$$

where $j_{0}$ is the least integer such that $2^{j_{0}} \geq \gamma \rho\left(x_{0}\right) / r$.

By Hölder's inequality and Lemma 10 , we obtain, for $j \leq$ $j_{0}$,

$$
\int_{2^{j} B}|f(y)|\left|b(y)-b_{B}\right| d y \leq j[b]_{\theta}\left|2^{j} B\right| \inf _{x \in B} M_{s} f(x) .
$$

Then,

$$
\begin{aligned}
I_{11} & \lesssim[b]_{\theta} \inf _{x \in B} M_{s} f(x) \sum_{j=2}^{j_{0}} j 2^{-j / 2} \\
& \lesssim[b]_{\theta_{x \in B} \inf _{s} M_{s} f(x) .}
\end{aligned}
$$

To deal with $I_{12}$, by using Lemma 10 and choosing $N>\theta^{\prime}$, we have

$$
\begin{aligned}
I_{12} & \leqslant \frac{\rho\left(x_{0}\right)^{N}}{r^{d+N}} \sum_{j=j_{0}-1}^{\infty} 2^{-j(d+1 / 2+N)} \int_{2^{j} B}|f(y)|\left|b(y)-b_{B}\right| d y \\
& \leqslant[b]_{\theta_{x \in B}} \inf _{s} f(x)\left(\frac{\rho\left(x_{0}\right)}{r}\right)^{N-\theta^{\prime}} \sum_{j=j_{0}-1}^{\infty} j 2^{-j\left(1 / 2+N-\theta^{\prime}\right)} \\
& \leqslant[b]_{\theta} \inf _{x \in B} M_{s} f(x) \sum_{j=j_{0}-1}^{\infty} j 2^{-j / 2} \\
& \leqslant[b]_{\theta} \inf _{x \in B} M_{s} f(x) .
\end{aligned}
$$


For $I_{3}$, again by Minkowski's inequality and in our situation $\rho(u) \simeq \rho\left(x_{0}\right)$ and $|u-y| \simeq|z-y|$, we get

$$
\begin{aligned}
I_{3} \lesssim & \int_{R^{d}}\left|f_{2}(y)\right|\left|b(y)-b_{B}\right|\left|K_{j}^{L}(u, y)-K_{j}^{L}(z, y)\right| \\
& \times\left(\int_{\{|u-y| \leq t,|z-y| \leq t\}} \frac{d t}{t^{3}}\right)^{1 / 2} d y \\
\leq & \int_{(2 B)^{c}} \frac{|f(y)|\left|b(y)-b_{B}\right|\left|K_{j}^{L}(u, y)-K_{j}^{L}(z, y)\right|}{|u-y|} d y \\
\leq & r^{\delta} \int_{Q \mid 2 B} \frac{|f(y)|\left|b(y)-b_{B}\right|}{|u-y|^{d+\delta}} d y \\
& +r^{\delta} \rho\left(x_{0}\right)^{N} \int_{Q^{c}} \frac{|f(y)|\left|b(y)-b_{B}\right|}{|u-y|^{d+\delta+N}} d y \\
= & I_{31}+I_{32} .
\end{aligned}
$$

Thanks to Hölder's inequality and Lemma 10, we have

$$
\begin{aligned}
I_{31} & \lesssim \frac{1}{r^{d}} \sum_{j=2}^{j_{0}} 2^{-j(d+\delta)} \int_{2^{j} B}|f(y)|\left|b(y)-b_{B}\right| d y \\
& \lesssim \frac{1}{r^{d}} \sum_{j=2}^{j_{0}} 2^{-j(d+\delta)} j[b]_{\theta}\left|2^{j} B\right| \inf _{x \in B} M_{s} f(x) \\
& \lesssim[b]_{\theta} \inf _{x \in B} M_{s} f(x) \sum_{j=2}^{j_{0}} j 2^{-j \delta} \\
& \lesssim[b]_{\theta} \inf _{x \in B} M_{s} f(x) .
\end{aligned}
$$

To deal with $I_{22}$, similarly as $I_{12}$, we have

$$
\begin{aligned}
I_{32} & \leqslant \frac{\rho\left(x_{0}\right)^{N}}{r^{d+N}} \sum_{j=j_{0}-1}^{\infty} 2^{-j(d+\delta+N)} \int_{2^{j} B}|f(y)|\left|b(y)-b_{B}\right| d y \\
& \leqslant[b]_{\theta} \inf _{x \in B} M_{s} f(x)\left(\frac{\rho\left(x_{0}\right)}{r}\right)^{N-\theta^{\prime}} \sum_{j=j_{0}-1}^{\infty} j 2^{-j\left(\delta+N-\theta^{\prime}\right)} \\
& \leq[b]_{\theta} \inf _{x \in B} M_{s} f(x) \sum_{j=j_{0}-1}^{\infty} j 2^{-j \delta} \\
& \leq[b]_{\theta} \inf _{x \in B} M_{s} f(x) .
\end{aligned}
$$

We have completed the proof of the lemma.

\section{The Boundedness of Marcikiewicz Integral and Its Commutator}

In this section, we first employ the same technique in [6] to prove Theorem 5 .
Proof. Similarly as [6], it suffices to prove the following pointwise estimate:

$$
\mu_{j}^{L} f(x) \leq \mu_{j} f(x)+C M f(x), \quad \text { a.e. } x \in R^{d},
$$

where $M$ denotes the Hardy-Littlewood operator.

Fix $x \in R^{d}$ and let $r=\rho(x)$. Notice that

$$
\begin{aligned}
\mu_{j}^{L} f(x) \leq & \left(\int_{0}^{r}\left|\int_{|x-y| \leq t} K_{j}^{L}(x, y) f(y) d y\right|^{2} \frac{d t}{t^{3}}\right)^{1 / 2} \\
& +\left(\int_{r}^{\infty}\left|\int_{|x-y| \leq r} K_{j}^{L}(x, y) f(y) d y\right|^{2} \frac{d t}{t^{3}}\right)^{1 / 2} \\
& +\left(\int_{r}^{\infty}\left|\int_{r<|x-y| \leq t} K_{j}^{L}(x, y) f(y) d y\right|^{2} \frac{d t}{t^{3}}\right)^{1 / 2} \\
\leq & \left(\int_{0}^{r}\left|\int_{|x-y| \leq t}\left[K_{j}^{L}(x, y)-K_{j}(x, y)\right] f(y) d y\right|^{2} \frac{d t}{t^{3}}\right)^{1 / 2} \\
& +\left(\int_{0}^{r}\left|\int_{|x-y| \leq t} K_{j}(x, y) f(y) d y\right|^{2} \frac{d t}{t^{3}}\right)^{1 / 2} \\
& +\left(\int_{r}^{\infty}\left|\int_{|x-y| \leq r} K_{j}^{L}(x, y) f(y) d y\right|^{2} \frac{d t}{t^{3}}\right)^{1 / 2} \\
& +\left(E_{1}^{\infty}\left|\int_{r<|x-y| \leq t} K_{j}^{L}(x, y) f(y) d y\right|^{2} \frac{d t}{t^{3}}\right)^{1 / 2}
\end{aligned}
$$

For $E_{1}$, by Lemma 4 , we have

$$
\begin{aligned}
E_{1} & \lesssim\left(\int_{0}^{r}\left|\int_{|x-y| \leq t} \frac{1}{|x-y|^{d-1}}\left(\frac{|x-y|}{\rho(x)}\right)^{\delta} f(y) d y\right|^{2} \frac{d t}{t^{3}}\right)^{1 / 2} \\
& \lesssim r^{-\sigma}\left(\int_{0}^{r}\left|\sum_{k=-\infty}^{0} \frac{1}{\left(2^{k-1} t\right)^{d-\delta-1}} \int_{|x-y| \leq 2^{k} t}\right| f(y)|d y|^{2} \frac{d t}{t^{3}}\right)^{1 / 2} \\
& \lesssim r^{-\sigma}\left(\int_{0}^{r}\left|\sum_{k=-\infty}^{0} \frac{\left(2^{k}\right)^{\delta+1} t^{\delta+1}}{\left(2^{k} t\right)^{d}} \int_{|x-y| \leq 2^{k} t}\right| f(y)|d y|^{2} \frac{d t}{t^{3}}\right)^{1 / 2} \\
& \leqslant r^{-\sigma}\left(\int_{0}^{r}\left|\sum_{k=-\infty}^{0}\left(2^{k}\right)^{\delta+1} t^{\delta+1} M f(x)\right|^{2} \frac{d t}{t^{3}}\right)^{1 / 2} \\
& \lesssim r^{-\sigma}\left(\int_{0}^{r} t^{2 \delta-1} d t\right)^{1 / 2} M f(x) \\
& \leqslant M f(x) .
\end{aligned}
$$


Obviously,

$$
E_{2} \leq \mu_{j} f(x) .
$$

For $E_{3}$, using Lemma 4 again, we get

$$
\begin{aligned}
E_{3} & \lesssim\left(\int_{r}^{\infty}\left|\int_{|x-y| \leq r} \frac{1}{|x-y|^{d-1}} f(y) d y\right|^{2} \frac{d t}{t^{3}}\right)^{1 / 2} \\
& \lesssim\left(\int_{r}^{\infty}\left|\sum_{k=-\infty}^{0} \frac{1}{\left(2^{k-1} r\right)^{d-1}} \int_{|x-y| \leq 2^{k} r} f(y) d y\right|^{2} \frac{d t}{t^{3}}\right)^{1 / 2} \\
& \leqslant\left(\int_{r}^{\infty}\left|\sum_{k=-\infty}^{0} \frac{2^{k} r}{\left(2^{k} r\right)^{d}} \int_{|x-y| \leq 2^{k} r} f(y) d y\right|^{2} \frac{d t}{t^{3}}\right)^{1 / 2} \\
& \lesssim\left(\int_{r}^{\infty}\left|\sum_{k=-\infty}^{0} 2^{k} r M f(x)\right|^{2} \frac{d t}{t^{3}}\right)^{1 / 2} \\
& \leqslant r\left(\int_{r}^{\infty} \frac{d t}{t^{3}}\right)^{1 / 2} M f(x) \\
& \leqslant M f(x) .
\end{aligned}
$$

It remains to estimate $E_{4}$. From Lemma 4 , we obtain

$$
\begin{aligned}
E_{4} & \lesssim\left(\int_{r}^{\infty}\left|r \int_{r<|x-y| \leq t} \frac{|f(y)|}{|x-y|^{n}} d y\right|^{2} \frac{d t}{t^{3}}\right)^{1 / 2} \\
& \lesssim r\left(\int_{r}^{\infty}\left|\sum_{k=0}^{\left[\log _{2} t / r\right]+1}\left(2^{k} r\right)^{-n} \int_{|x-y| \leq 2^{k} r} f(y) d y\right|^{2} \frac{d t}{t^{3}}\right)^{1 / 2} \\
& \lesssim r\left(\int_{r}^{\infty}\left|\left(\left[\log _{2} \frac{t}{r}\right]+1\right) M f(x)\right|^{2} \frac{d t}{t^{3}}\right)^{1 / 2} \\
& \lesssim r\left(\int_{r}^{\infty} \frac{t}{r} M f(x)^{2} \frac{d t}{t^{3}}\right)^{1 / 2} \\
& \lesssim M f(x) .
\end{aligned}
$$

With the help of the $L^{p}$-boundedness and weak $(1,1)$ boundedness of $\mu_{j}$ and $M$, we can get the same boundedness of $\mu_{j}^{L}$.

For the $\mathrm{BMO}_{L}$-boundedness and $\left(H_{L}^{1}, L^{1}\right)$-boundedness of $\mu_{j}^{L}$, we only make similar modification in the procedure of the same estimate in [6]. Here, we omit it.

Next we will establish some boundedness for the commutator of Marcinkiewicz integral of Schrödinger operator type.

We start with the proof of Theorem 6.

Proof. For any function $f \in L^{p}\left(R^{d}\right)$ with $1<p<p_{0}$, we notice that, due to Lemma 11 , we have $\left[b, \mu_{j}^{L}\right] f \in L_{\text {loc }}^{1}\left(R^{d}\right)$.
By using Lemma 8 and Lemma 11 with $1<p<p_{0}$, and Remark 12, we have

$$
\begin{aligned}
\left\|\left[b, \mu_{j}^{L}\right] f\right\|_{p}^{p} \leq & \int_{R^{d}}\left|M_{\rho, \beta}\left(\left[b, \mu_{j}^{L}\right] f\right)\right|^{p} \\
\leq & \int_{R^{d}}\left|M_{\rho, \gamma}^{\sharp}\left(\left[b, \mu_{j}^{L}\right] f\right)\right|^{p} \\
& +\sum_{k}\left|Q_{k}\right|\left(\frac{1}{\left|Q_{k}\right|} \int_{2 Q_{k}}\left|\left[b, \mu_{j}^{L}\right] f\right|\right)^{p} \\
\lesssim & \int_{R^{d}}\left|M_{\rho, \gamma}^{\sharp}\left(\left[b, \mu_{j}^{L}\right] f\right)\right|^{p}+[b]_{\theta}^{p} \sum_{k} \int_{2 Q_{k}}\left|M_{s} f\right|^{p} .
\end{aligned}
$$

By the finite overlapping property given by Proposition 2 and the boundedness of $M_{s}$ in $L^{p}\left(R^{d}\right)$, the second term is controlled by $[b]_{\theta}^{p}\|f\|_{p}^{p}$. Thus, we have to take care of the first term.

Our goal is to find a point-wise estimate of $M_{\rho, \gamma}^{\sharp}\left(\left[b, \mu_{j}^{L}\right] f\right)$. Let $x \in R^{d}$ and $B=B\left(x_{0}, r\right)$, with $r<\gamma \rho\left(x_{0}\right)$ such that $x \in B$. If $f=f_{1}+f_{2}$, with $f_{1}=f \chi_{2 B}$, then we write

$$
\begin{aligned}
\frac{1}{|B|} \int_{B}\left[b, \mu_{j}^{L}\right] f(x) d x \leq & \frac{1}{|B|} \int_{B}\left|b-b_{B}\right| \mu_{j}^{L} f(x) d x \\
& +\frac{1}{|B|} \int_{B} \mu_{j}^{L}\left(f_{1}\left(b-b_{B}\right)\right)(x) d x \\
& +\frac{1}{|B|} \int_{B} \mu_{j}^{L}\left(f_{2}\left(b-b_{B}\right)\right)(x) d x \\
= & U_{1}+U_{2}+U_{3} .
\end{aligned}
$$

Let $1<s<p_{0}$; an application of Hölder's inequality and Lemma 10 gives

$$
\begin{aligned}
U_{1} & =\frac{1}{|B|} \int_{B}|| b-b_{B}\left|\mu_{j}^{L} f-\frac{1}{|B|} \int_{B}\right| b-b_{B}\left|\mu_{j}^{L} f\right| \\
& \leq \frac{2}{|B|} \int_{B}\left|\left(b-b_{B}\right) \mu_{j}^{L} f\right| \\
& \leq\left(\frac{1}{|B|} \int_{B}\left|b-b_{B}\right|^{s^{\prime}}\right)^{1 / s^{\prime}}\left(\frac{1}{|B|} \int_{B}\left|\mu_{j}^{L} f\right|^{s}\right)^{1 / s} \\
& \leq[b]_{\theta} M_{s} \mu_{j}^{L} f(x),
\end{aligned}
$$

since $r / \rho\left(x_{0}\right)<\gamma$. 
To estimate $U_{2}$, let $1<\widetilde{s}<s<p_{0}$ and $v=\widetilde{s} s /(s-\widetilde{s})$. Then,

$$
\begin{aligned}
U_{2} & =\frac{1}{|B|} \int_{B}\left|\mu_{j}^{L}\left(\left(b-b_{B}\right) f_{1}\right)-\frac{1}{|B|} \int_{B} \mu_{j}^{L}\left(\left(b-b_{B}\right) f_{1}\right)\right| \\
& \leq \frac{2}{|B|} \int_{B}\left|\mu_{j}^{L}\left(\left(b-b_{B}\right) f_{1}\right)\right| \\
& \leq\left(\frac{1}{|B|} \int_{B}\left|\mu_{j}^{L}\left(\left(b-b_{B}\right) f_{1}\right)\right|^{\widetilde{s}}\right)^{1 / \widetilde{s}} \\
& \leq\left(\frac{1}{|B|} \int_{2 B}\left|\left(b-b_{B}\right) f\right|^{\widetilde{s}}\right)^{1 / \widetilde{s}} \\
& \leq\left(\frac{1}{|B|} \int_{2 B}\left|b-b_{B}\right|^{\nu}\right)^{1 / v}\left(\frac{1}{|B|} \int_{2 B}|f|^{s}\right)^{1 / s} \\
& \leq[b]_{\theta} M_{s} f(x) .
\end{aligned}
$$

For $U_{3}$, using Lemma 13, we get

$$
\begin{aligned}
U_{3} & =\frac{1}{|B|} \int_{B} \mid \mu_{j}^{L}\left(f_{2}\left(b-b_{B}\right)\right)(u) \\
& \quad-\frac{1}{|B|} \int_{B} \mu_{j}^{L}\left(f_{2}\left(b-b_{B}\right)\right)(z) \mid d u d z \\
\leq & \frac{1}{|B|^{2}} \iint_{B} \mid \mu_{j}^{L}\left(f_{2}\left(b-b_{B}\right)\right)(u) \\
& \quad-\mu_{j}^{L}\left(f_{2}\left(b-b_{B}\right)\right)(z) \mid d u d z \\
& \leq \frac{1}{|B|^{2}} \iint_{B}[b]_{\theta} M_{s} f(x) d u d z \\
& \leq[b]_{\theta} M_{s} f(x) .
\end{aligned}
$$

Therefore, we have proved that

$$
\left|M_{\rho, \gamma}^{\sharp}\left(\left[b, \mu_{j}^{L}\right] f\right)\right| \lesssim[b]_{\theta}\left[M_{s} \mu_{j}^{L} f(x)+M_{s} f(x)\right] .
$$

Since $s<p$, we obtain the desired result.

Finally, we will prove Theorem 7.

Proof. Let $f \in L^{\infty}\left(R^{d}\right)$ and $Q=B\left(x_{0}, \gamma \rho\left(x_{0}\right)\right)$. From Proposition 2, we only consider averages over critical ball. Thanks to Lemma 11, one gets

$$
\frac{1}{|Q|} \int_{Q}\left|\left[b, \mu_{j}^{L}\right] f\right| \lesssim[b]_{\theta} \inf _{y \in Q} M_{s} f(y) \lesssim[b]_{\theta}\|f\|_{\infty} .
$$

Next, we deal with the oscillations; let $B=B\left(x_{0}, r\right)$ with $r<\gamma \rho\left(x_{0}\right)$. Lemma 11 states that the function $\left[b, \mu_{j}^{L}\right] f$ belongs to $L_{\text {loc }}^{1}\left(R^{d}\right)$.
We write

$$
\begin{aligned}
& {\left[b, \mu_{j}^{L}\right] f} \\
& \quad \leq\left|b-b_{B}\right| \mu_{j}^{L} f+\mu_{j}^{L}\left(f_{1}\left(b-b_{B}\right)\right)+\mu_{j}^{L}\left(f_{2}\left(b-b_{B}\right)\right),
\end{aligned}
$$

and its mean oscillations on $B$ as $E_{1}, E_{2}$, and $E_{3}$.

The estimates for the terms $E_{2}$ and $E_{3}$ are the same with $U_{2}$ and $U_{3}$ in Theorem 6. Due to the boundedness of $\mu_{j}^{L}$ in $L^{\widetilde{s}}\left(R^{d}\right)$, for $\widetilde{s}>1$, and Lemma 13 , we get that $E_{2}$ and $E_{3}$ are bounded by $[b]_{\theta}\|f\|_{\infty}$.

To deal with $E_{1}$, we fix $u \in B$ and write

$$
\begin{aligned}
\left|b-b_{B}\right| \mu_{j}^{L} f \leq & \left|b-b_{B}\right| \mu_{j}^{L} f_{1}+\left|b-b_{B}\right|\left(\mu_{j}^{L} f_{2}-\mu_{j}^{L} f_{2}(u)\right) \\
& +\mu_{j}^{L} f_{21}(u)\left|b-b_{B}\right|+\mu_{j}^{L} f_{22}(u)\left|b-b_{B}\right|,
\end{aligned}
$$

where $f_{2}=f_{21}+f_{22}$, with $f_{22}=f \chi_{4 Q \mid 2 B}$ and $Q=$ $B\left(x_{0}, \gamma \rho\left(x_{0}\right)\right)$. We denoted each oscillation $E_{11}, E_{12}, E_{13}$, and $E_{14}$.

We observe that $\mu_{j}^{L} f_{21}(u)$ and $\mu_{j}^{L} f_{22}(u)$ are finite, for any $u \in B$, since $f \in L^{\infty}$ and

$$
\int_{(2 B)^{c}}\left|K_{j}^{L}(u, z)\right| d z<\infty
$$

We will see that $E_{11}, E_{12}$, and $E_{13}$ are bounded under the condition $b \in \mathrm{BMO}_{\infty}(\rho)$.

For $E_{11}$, by choosing $s$ such that $\mu_{j}^{L}$ is bounded on $L^{s}\left(R^{d}\right)$, we have

$$
\begin{aligned}
E_{11} & \leq \frac{2}{|B|} \int_{B}\left|\left(b-b_{B}\right) \mu_{j}^{L} f_{1}\right| \\
& \leq\left(\frac{1}{|B|} \int_{B}\left|b-b_{B}\right|^{s^{\prime}}\right)^{1 / s^{\prime}}\left(\frac{1}{|B|} \int_{B}\left|\mu_{j}^{L} f_{1}\right|^{s}\right)^{1 / s} \\
& \leq\left(\frac{1}{|B|} \int_{B}\left|b-b_{B}\right|^{s^{\prime}}\right)^{1 / s^{\prime}}\left(\frac{1}{|B|} \int_{2 B}|f|^{s}\right)^{1 / s} \\
& \leq[b]_{\theta}\|f\|_{\infty} .
\end{aligned}
$$

To deal with $E_{12}$, we claim

$$
\left|\mu_{j}^{L} f_{2}(x)-\mu_{j}^{L} f_{2}(u)\right| \lesssim\|f\|_{\infty},
$$

for any $x$ and $u$ in $B$. 
It is easy to see that

$$
\begin{aligned}
\mid \mu_{j}^{L} f_{2}(x) & -\mu_{j}^{L} f_{2}(u) \mid \\
\leq & \left(\int_{0}^{\infty}\left|\int_{|x-y| \leq t<|u-y|}\right| K_{j}^{L}(x, y) f_{2}(y)|d y|^{2} \frac{d t}{t^{3}}\right)^{1 / 2} \\
& +\left(\int_{0}^{\infty}\left|\int_{|u-y| \leq t<|x-y|}\right| K_{j}^{L}(u, y) f_{2}(y)|d y|^{2} \frac{d t}{t^{3}}\right)^{1 / 2} \\
& +\left(\int_{0}^{\infty}\left|\int_{\{|x-y| \leq t,|u-y| \leq t\}}\right| K_{j}^{L}(x, y)-K_{j}^{L}(u, y) \mid\right. \\
= & I_{1}+I_{2}+I_{3} .
\end{aligned}
$$

Since the estimates for $I_{1}$ and $I_{2}$ follow along similar lines, we only consider $I_{1}$. Since $|x-y| \simeq|u-y|$, by Minkowski's inequality and Lemma 4 , we have

$$
\begin{aligned}
I_{1} & \lesssim \int_{R^{n}}\left|f_{2}(y)\right|\left|K_{j}^{L}(x, y)\right|\left(\int_{|x-y| \leq t<|u-y|} \frac{d t}{t^{3}}\right)^{1 / 2} d y \\
& \lesssim r^{1 / 2} \int_{(2 B)^{c}} \frac{|f(y)|\left|K_{j}^{L}(x, y)\right|}{|x-y|^{3 / 2}} d y \\
& \lesssim r^{1 / 2} \int_{(2 B)^{c}} \frac{|f(y)|}{|x-y|^{n+1 / 2}} d y \\
& \lesssim \frac{1}{r^{n}} \sum_{j=1}^{\infty} 2^{-j(n+1 / 2)} \int_{2^{j+1} B}|f(y)| d y \\
& \leq\|f\|_{\infty} .
\end{aligned}
$$

For $I_{3}$, again thanks to the Minkowski's inequality, Lemma 4 , and $|x-y|=|u-y|$, we obtain

$$
\begin{aligned}
I_{3} & \lesssim \int_{R^{n}}\left|f_{2}(y)\right|\left|K_{j}^{L}(x, y)-K_{j}^{L}(u, y)\right| \\
& \times\left(\int_{\{|x-y| \leq t,|u-y| \leq t\}} \frac{d t}{t^{3}}\right)^{1 / 2} d y \\
& \lesssim \int_{(2 B)^{c}} \frac{|f(y)|\left|K_{j}^{L}(x, y)-K_{j}^{L}(u, y)\right|}{|x-y|} d y \\
& \leq r^{\delta} \int_{(2 B)^{c}} \frac{|f(y)|}{|x-y|^{n+\delta}} d y \\
& \leq \frac{1}{r^{n}} \sum_{j=1}^{\infty} 2^{-j(n+\delta)} \int_{2^{j+1} B}|f(y)| d y \\
& \leq\|f\|_{\infty} .
\end{aligned}
$$

Thus, the claim is completed.
Then,

$$
\begin{aligned}
E_{12} & \leq \frac{2}{|B|} \int_{B}\left|b-b_{B}\right|\left|\mu_{j}^{L} f_{2}(x)-\mu_{j}^{L} f_{2}(u)\right| d x \\
& \lesssim[b]_{\theta}\|f\|_{\infty} .
\end{aligned}
$$

That $E_{13} \lesssim[b]_{\theta}\|f\|_{\infty}$ is a consequence of (54).

Therefore, the theorem will follow, if there exists a constant $C$ such that, for any $B \in \mathbf{B}_{\rho, \gamma}$ and $u \in B$,

$$
\left(\frac{1}{|B|} \int_{B}\left|b(y)-b_{B}\right| d y\right)\left|\mu_{j}^{L} f_{22}(u)\right| \leq C\|b\|_{* \log }\|f\|_{\infty} .
$$

Using Minkowski's inequality,

$$
\begin{aligned}
\left|\mu_{j}^{L} f_{22}(u)\right| & =\left(\int_{0}^{\infty}\left|\int_{|x-y| \leq t} K_{j}^{L}(x, y) f_{22}(y) d y\right|^{2} \frac{d t}{t^{3}}\right)^{1 / 2} \\
& \leq \int_{R^{d}}\left|\frac{f_{22}(y)}{|u-y|^{d-1}}\right|\left(\int_{|u-y| \leq t} \frac{d t}{t^{3}}\right)^{1 / 2} d y \\
& \leq \int_{4 \mathrm{Q} \backslash 2 B} \frac{|f(y)|}{|u-y|^{d}} d y \\
& \leq\|f\|_{\infty} \log \frac{\rho\left(x_{0}\right)}{r} .
\end{aligned}
$$

Since $b \in \operatorname{BMO}_{\infty}^{\log }(\rho)$, we conclude that (61) holds proving the boundedness of $\left[b, \mu_{j}^{L}\right]$.

\section{Conflict of Interests}

The authors declare that there is no conflict of interests regarding the publication of this paper.

\section{Acknowledgments}

The authors would like to express their heartfelt thanks to the referee and Professor Yong Sheng Han's suggestions. This work is supported by the National Natural Science Foundation of China (10961015, 11261023, and 11326092) and the Jiangxi Natural Science Foundation of China (20122BAB201011). The foundation of Jiangxi teaching devision GJJ12203.

\section{References}

[1] E. Stein, "On the functions of Littlewood-Paley, Lusin, and Marcinkiewicz," Transactions of the American Mathematical Society, vol. 88, pp. 430-466, 1958.

[2] A. Benedek, A. P. Calderón, and R. Panzone, "Convolution operators on Banach space valued functions," Proceedings of the National Academy of Sciences of the United States of America, vol. 48, pp. 356-365, 1962.

[3] Y. Ding, S. Lu, and Q. Xue, "Marcinkiewicz integral on hardy spaces," Integral Equations and Operator Theory, vol. 42, no. 2, pp. 174-182, 2002. 
[4] Z. Shen, " $L^{p}$ estimates for Schrödinger operators with certain potentials," Annales de l'institut Fourier, vol. 45, no. 2, pp. 513546, 1995.

[5] F. W. Gehring, "The $L^{p}$-integrability of the partial derivatives of a quasiconformal mapping," Acta Mathematica, vol. 130, no. 1, pp. 265-277, 1973.

[6] L. Tang and J. F. Dong, "Boundedness for Marcinkiewicz integrals associatedwith Schrödinger operators," preprint.

[7] J. Dziubański, G. Garrigós, T. Martínez, J. L. Torrea, and J. Zienkiewicz, "BMO spaces related to Schrödinger operators with potentials satisfying a reverse Hölder inequality," Mathematische Zeitschrift, vol. 249, no. 2, pp. 329-356, 2005.

[8] C. Fefferman and E. M. Stein, " $H^{p}$ spaces of several variables," Acta Mathematica, vol. 129, no. 1, pp. 137-193, 1972.

[9] B. Bongioanni, E. Harboure, and O. Salinas, "Commutators of Riesz transforms related to Schrödinger operators," Journal of Fourier Analysis and Applications, vol. 17, no. 1, pp. 115-134, 2011. 


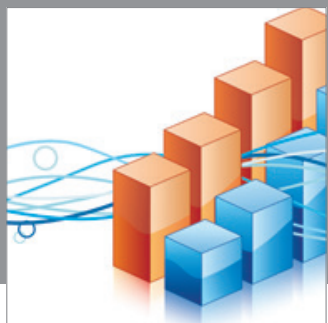

Advances in

Operations Research

mansans

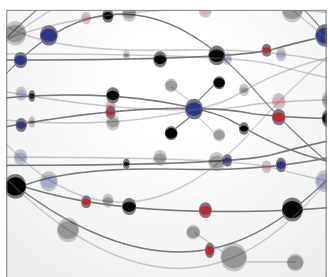

The Scientific World Journal
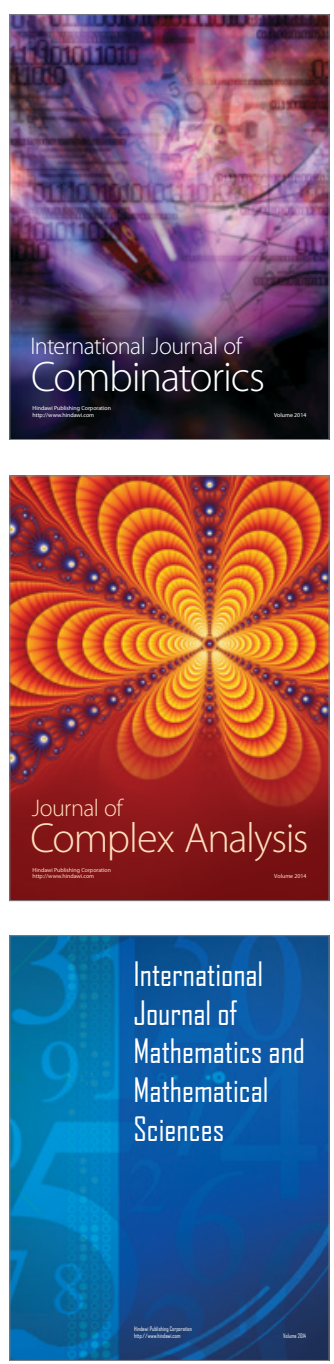
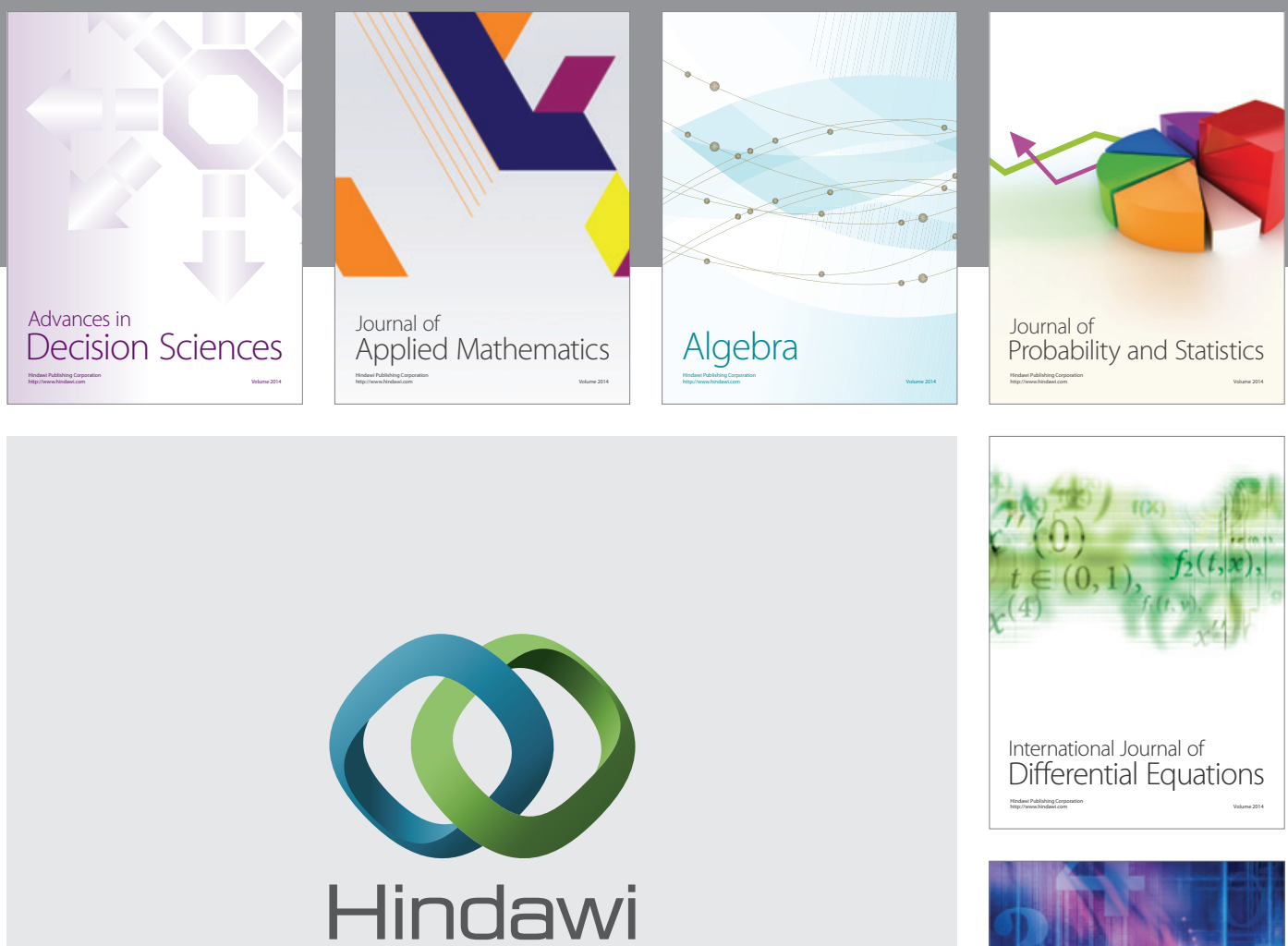

Submit your manuscripts at http://www.hindawi.com
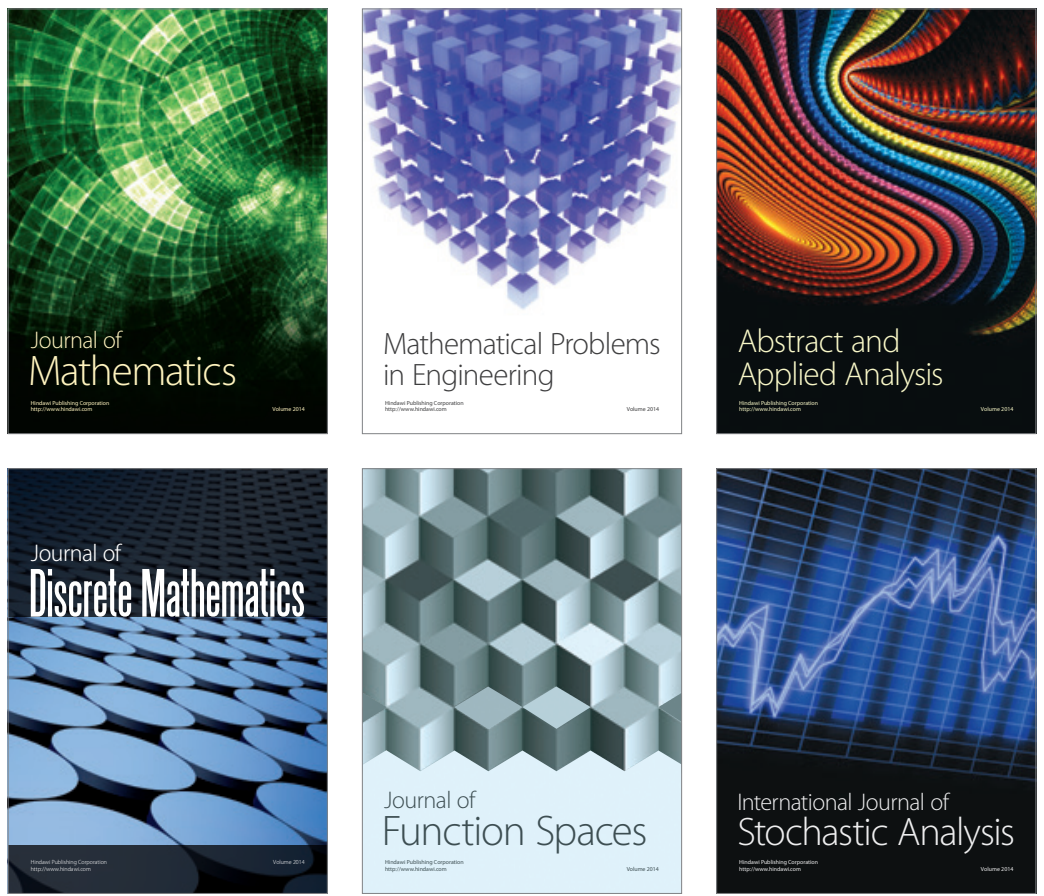

Journal of

Function Spaces

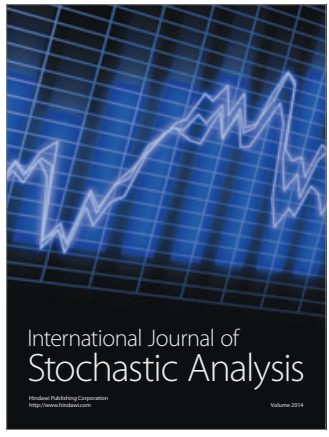

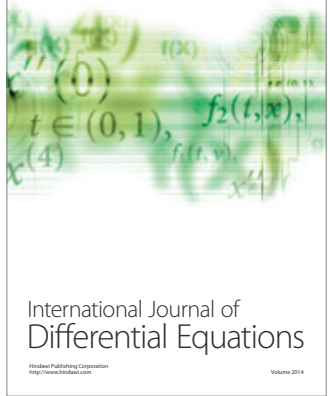
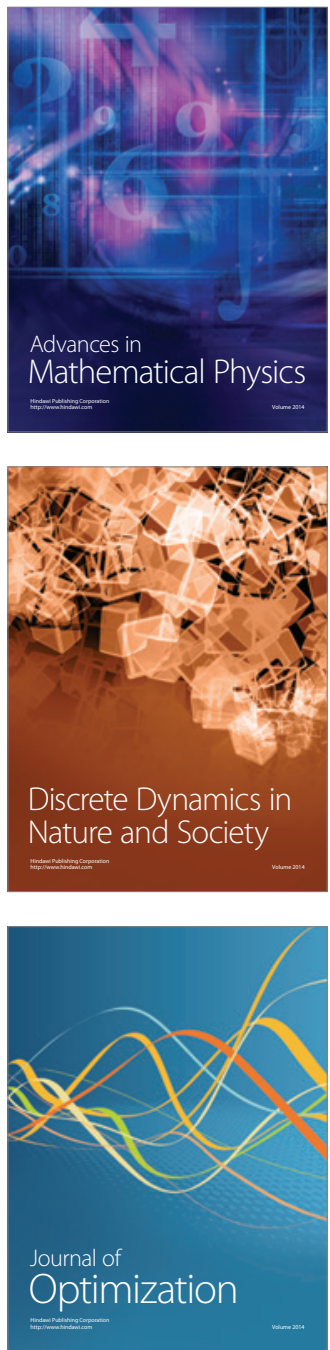